\title{
CLASSICAL SOLUTIONS OF THE HAMILTON-JACOBI-BELLMAN EQUATION FOR UNIFORMLY ELLIPTIC OPERATORS
}

BY

\author{
LAWRENCE C. EVANS
}

\begin{abstract}
We prove under appropriate hypotheses that the Hamilton-JacobiBellman dynamic programming equation with uniformly elliptic operators, $\max _{1<k<m}\left\{L^{k} u-f^{k}\right\}=0$, has a classical solution $u \in C^{2, \beta}$, for some (small) Hölder exponent $\beta>0$.
\end{abstract}

1. Introduction. This paper extends some methods developed in [4] to prove the existence and uniqueness of a classical solution $u$ of the Hamilton-Jacobi-Bellman equation:

$$
\begin{cases}\max _{1 \leqslant k \leqslant m}\left\{L^{k} u(x)-f^{k}(x)\right\}=0, & x \in \Omega, \\ u(x)=0 & x \in \partial \Omega .\end{cases}
$$

Here $\Omega$ denotes a bounded, smooth domain in $\mathbf{R}^{n}(n \geqslant 1)$, the $f^{k}$ are given smooth functions, and the $L^{k}$ are second order, uniformly elliptic operators of the form

$$
L^{k} u \equiv a_{i j}^{k}(x) u_{x_{i} x_{j}}+b_{i}^{k}(x) u_{x_{i}}+c^{k}(x) u,
$$

with smooth coefficients satisfying

$$
\begin{cases}c^{k}(x) \leqslant 0, & x \in \bar{\Omega}, \\ \theta|\xi|^{2} \leqslant a_{i j}^{k}(x) \xi_{i} \xi_{j} \leqslant \Theta|\xi|^{2}, & x \in \bar{\Omega}, \xi \in \mathbf{R}^{n},\end{cases}
$$

for constants $0<\theta \leqslant \Theta<\infty, k=1, \ldots, m$.

Problem (1.1) is the equation of dynamic programming arising in the study of the optimal control of certain diffusion processes. For an explanation of its formal derivation from control theory principles see Krylov [9], Bensoussan-J. L. Lions [2], Fleming-Rishel [7], or Kushner [13]. In this interpretation $L^{k}$ describes in some sense the response of the stochastic system under control to the constant control $k, f^{k}$ denotes the running cost of control $k$, and $u(x)$ is the value function, i.e., the (expected) payoff for the optimally controlled system starting in state $x$.

Received by the editors May 18, 1981 and, in revised form, December 4, 1981.

1980 Mathematics Subject Classification. Primary 35J60, 93E20.

Key words and phrases. Nonlinear elliptic p.d.e., a priori estimate, dynamic programming.

(C) 1983 American Mathematical Society 0002-9947/82/0000-0391/\$03.75 
The references just mentioned all provide calculations showing that should the value function $u$ be smooth enough (say $C^{2}$ ), then it satisfies (1.1); and-conversely - if (1.1) has a smooth enough solution it must be the value function. This second fact motivates a direct study of (1.1), in analogy with the indirect methods of the calculus of variations. Many papers in the last few years have made rigorous the formal calculations of dynamic programming theory by demonstrating under assorted hypotheses the existence of a (unique) solution with various regularity properties: see Krylov [9-10], Nisio [19], Brezis-Evans [3], Evans-Friedman [5], P. L. Lions-Menaldi [18], P. L. Lions [16-17], Evans-P. L. Lions [6], Belbas [1], etc. Under the assumption (1.3) of uniform ellipticity the best results to date were in [6], where we proved (1.1) has a unique solution $u \in W^{2, \infty}(\Omega)$, solving (1.1) for a.e. $x \in \Omega$.

In this work we will show that the solution of (1.1) is in fact a classical solution, lying in the space $C_{\mathrm{loc}}^{2, \beta}$ for some perhaps very small Hölder exponent $\beta>0$. (This was proved in [3] for the case $m=2$ by other, much simpler methods.) The main ideas for the proof are taken from a recent paper [4], where we in fact already obtained this result for the (unrealistic) situation that the operators $L^{k}$ have constant coefficients, with no lower order terms. The key observation there was that any pure second derivative $v \equiv u_{\xi \xi}$ ( $\xi$ a unit vector) is a supersolution of a certain linear elliptic p.d.e., to which some recent estimates of Krylov-Safonov [11] apply. In the general situation that the coefficients of the $L^{k}$ depend on $x, v=u_{\xi \xi}$ is unfortunately no longer a supersolution.

Happily it turns out, as we will show in this paper, that a relatively small modification of $v=u_{\xi \xi}$ in fact does act like a supersolution; this technique we borrow from the proof of Ladyženskaja-Ural'ceva [14,p. 340] (see also GilbargTrudinger [8, p. 270]) concerning $C^{1, \alpha}$ estimates for quasilinear elliptic equations. With these calculations in hand it is then relatively routine to modify the methods of [4] to obtain an interior a priori estimate on the Hölder modulus of continuity of $D^{2} u$.

And once we have such bounds it is then straightforward to prove:

THEOREM 1. Under the hypotheses described above (1.1) has a unique solution $u \in C(\bar{\Omega}) \cap C^{2, \beta}(\Omega)$ for some $\beta>0$.

Note carefully we do not assert $u \in C^{2, \beta}(\bar{\Omega})$. Indeed it is an interesting open problem to extend the techniques of [4] to yield boundary estimates for the Hölder continuity of $D^{2} u$. Such bounds would also be useful for studying the Monge-Ampere equation.

The paper is organized so that the proof of Theorem 1 appears in $\S 4$, after a derivation in $\$ \S 2,3$ of the key estimate (Lemma 3.3) concerning the oscillation of $D^{2} u$ on concentric balls in $\Omega$. As noted previously the principal new ideas concern adapting the methods of [4] to cover the variable coefficient case: as such we will devote most of our attention to the new essential difficulties and will continually refer the reader back to [4] for those aspects of the proof which are not much changed. (See the "Note added in proof" at the end of $\$ 4$.) 
Notation.

$$
\begin{gathered}
D u \equiv\left(u_{x_{1}}, \ldots, u_{x_{n}}\right), \quad D^{2} u \equiv\left(u_{x_{1} x_{1}}, \ldots, u_{x_{i} x_{j}}, \ldots\right), \\
D^{3} u \equiv\left(\ldots, u_{x_{i} x_{j} x_{k}}, \ldots\right), \\
B\left(x_{0}, R\right) \equiv\left\{y \in \mathbf{R}^{n}|| x_{0}-y \mid \leqslant R\right\}, \quad B(R)=B(0, R), \\
e_{i} \equiv i \text { th unit vector in } \mathbf{R}^{n}, \\
{[f]_{C^{\beta}(K)} \equiv \max _{\substack{x, y \in K \\
x \neq y}} \frac{|f(x)-f(y)|}{|x-y|^{\beta}} .}
\end{gathered}
$$

We employ the summation convention throughout this paper; the letter " $C$ " denotes various constants depending only on known quantities.

2. Approximation; construction of supersolutions. The key to everything else in the proof of Theorem 1 is an a priori bound on the oscillation of $D^{2} u$ in $\Omega^{\prime}, \Omega^{\prime} \subset \subset \Omega$. We present in this section the necessary major modifications of the argument in [4], and will in fact derive the estimates for a sequence of approximating problems defined as follows.

According to [4, Lemma 7.3] there exist smooth functions $\beta^{\varepsilon}: \mathbf{R}^{m} \rightarrow \mathbf{R}(\varepsilon>0)$ satisfying

$$
\begin{cases}\text { (i) } & \beta^{\varepsilon}(\cdot) \text { is smooth and convex }(\varepsilon>0) \\ \text { (ii) } & 0 \leqslant \beta_{r_{i}}^{\varepsilon}(r) \leqslant 1, \sum_{i=1}^{m} \beta_{r_{i}}^{\varepsilon}(r)=1\left(\varepsilon>0, i=1, \ldots, m, r \in \mathbf{R}^{m}\right), \\ \text { (iii) } & \beta^{\varepsilon}(r)=\beta^{\varepsilon}\left(r_{1}, \ldots, r_{m}\right) \rightarrow \max _{1 \leqslant k \leqslant m} r_{k} \text { as } \varepsilon \succ 0 \\ & \text { uniformly on compact subsets of } \mathbf{R}^{m} \\ \text { (iv) } & \beta^{\varepsilon}\left(r_{0}, \ldots, r_{0}\right)=r_{0} \text { for each } r_{0} \in \mathbf{R}^{1}\end{cases}
$$

Let us therefore approximate (1.1) by the problem

$$
\begin{cases}\beta^{\varepsilon}\left(L^{1} u^{\varepsilon}(x)-f^{1}(x), \ldots, L^{m} u^{\varepsilon}(x)-f^{m}(x)\right)=0, & x \in \Omega, \\ u^{\varepsilon}(x)=0, & x \in \partial \Omega .\end{cases}
$$

For the remainder of this section we will suppose that $(2.2)_{\varepsilon}$ has a smooth solution $u^{\varepsilon}$ and then derive estimates on the oscillation of $D^{2} u^{\varepsilon}$, provided

$$
\max _{x \in \Omega}\left|u^{\varepsilon}\right|,\left|D u^{\varepsilon}\right|,\left|D^{2} u^{\varepsilon}\right| \leqslant M
$$

for some constant $M$, independent of $\varepsilon$. Later, in $\S 4$, we will make use of these bounds to prove the existence of a solution of $(2.2)_{\varepsilon}$ and then to pass to limits as $\varepsilon \searrow 0$ to solve (1.1).

So now let $\xi$ denote some unit vector in $\mathbf{R}^{n}$ and set

$$
v^{\varepsilon} \equiv u_{\xi \xi}^{\varepsilon}-\eta \sum_{i, j=1}^{n}\left(u_{x_{i} x_{j}}^{\varepsilon}-n^{2} M \delta_{i j}\right)^{2}
$$

$\eta$ denoting here some fixed positive number, which will be selected later (in $\S 3$ ). 
Define also

$$
a_{i j}^{\varepsilon}(x) \equiv \beta_{r_{k}}^{\varepsilon}(\cdots) a_{i j}^{k}(x) \quad(1 \leqslant i, j \leqslant n),
$$

and notice that according to (2.1)(ii) and (1.3),

$$
\theta|\xi|^{2} \leqslant a_{i j}^{\varepsilon}(x) \xi_{i} \xi_{j} \leqslant \Theta|\xi|^{2} \quad \text { for all } \xi \in \mathbf{R}^{n}, x \in \bar{\Omega} \text {. }
$$

LEMMA 2.1. There exists a constant $C(\eta)$ such that

$$
-a_{k l}^{\varepsilon} v_{x_{k} x_{l}}^{\varepsilon} \geqslant-C(\eta) \text { in } \Omega
$$

$C(\eta)$ depends only on $\eta, M, n$, the coefficients of the $L^{k}$, the $f^{k}$, and not on $\varepsilon$ or $\xi$.

Proof. To simplify the notation we drop the superscript " $\varepsilon$ ". Let us compute

$$
\begin{aligned}
v_{x_{k}} & =u_{\xi \xi x_{k}}-2 \eta\left(u_{x_{i} x_{j}}-n^{2} M \delta_{i j}\right) u_{x_{i} x_{j} x_{k}} \\
v_{x_{k} x_{l}} & =u_{\xi \xi x_{k} x_{l}}-2 \eta u_{x_{i} x_{j} x_{l}} u_{x_{i} x_{j} x_{k}}-2 \eta\left(u_{x_{i} x_{j}}-n^{2} M \delta_{i j}\right) u_{x_{i} x_{j} x_{k} x_{l}}
\end{aligned}
$$

Next differentiate (2.2) $)_{\varepsilon}$ with respect to $x_{i}$ and $x_{j}$, and also twice with respect to $\xi$ :

$$
\begin{gathered}
\beta_{r_{s}}(\cdot)\left(L^{s} u-f^{s}\right)_{x_{i} x_{j}}+\beta_{r_{s} r_{t}}(\cdot)\left(L^{s} u-f^{s}\right)_{x_{i}}\left(L^{t} u-f^{t}\right)_{x_{j}}=0 \\
\beta_{r_{s}}(\cdot)\left(L^{s} u-f^{s}\right)_{\xi \xi}+\beta_{r_{s} r_{t}}(\cdot)\left(L^{s} u-f^{s}\right)_{\xi}\left(L^{t} u-f^{t}\right)_{\xi}=0 .
\end{gathered}
$$

In view of the convexity of $\beta$, the last equation implies

$$
-\beta_{r_{s}}(\cdot)\left(L^{s} u-f^{s}\right)_{\xi \xi} \geqslant 0 .
$$

Now observe that (2.3), (2.8) and (2.9) yield

$$
-\beta_{r_{s}}(\cdot) a_{k l}^{s} u_{x_{i} x_{j} x_{k} x_{l}} \geqslant \beta_{r_{s} r_{t}}(\cdot)\left(L^{s} u-f^{s}\right)_{x_{i}}\left(L^{t} u-f^{t}\right)_{x_{j}}-C-C\left|D^{3} u\right|
$$

and

$$
-\beta_{r_{s}}(\cdot) a_{k l}^{s} u_{\xi \xi x_{k} x_{l}} \geqslant-C-C\left|D^{3} u\right| .
$$

Finally let us calculate

$$
\begin{aligned}
-a_{k l} v_{x_{k} x_{l}}= & -\beta_{r_{s}}(\cdot) a_{k l}^{s} v_{x_{k} x_{l}}=-\beta_{r_{s}}(\cdot) a_{k l}^{s} u_{\xi \xi x_{k} x_{l}}+2 \eta \beta_{r_{s}}(\cdot) a_{k l}^{s} u_{x_{i} x_{j} x_{l}} u_{x_{i} x_{j} x_{k}} \\
& -2 \eta\left(u_{x_{i} x_{j}}-n^{2} M \delta_{i j}\right)\left(-\beta_{r_{s}}(\cdot) a_{k l}^{s} u_{x_{i} x_{j} x_{k} x_{l}}\right) \\
\geqslant & -C-C\left|D^{3} u\right|+2 \eta \theta\left|D^{3} u\right|^{2}
\end{aligned}
$$

the last estimate results from (2.11), (2.6) and (2.10), respectively. Notice also that we have used here the inequality

$$
-2 \eta\left(u_{x_{i} x_{j}}-n^{2} M \delta_{i j}\right) \beta_{r_{s} r_{t}}(\cdot)\left(L^{s} u-f^{s}\right)_{x_{i}}\left(L^{t} u-f^{t}\right)_{x_{j}} \geqslant 0,
$$

resulting from the convexity of $\beta$ and the matrix inequality

$$
\left(\left(u_{x_{i} x_{j}}-n^{2} M \delta_{i j}\right)\right) \leqslant 0 .
$$

The lemma is an immediate consequence of (2.12).

Lemma 2.1 will be useful because of the following important estimate of KrylovSafonov [11]. Let us assume now that the ball $B\left(R_{0}\right)$ lies in $\Omega$ for some $R_{0}>0$. 
Define for $0<R<R_{0}$

$$
m^{\varepsilon}(R) \equiv \min _{B(R)} v^{\varepsilon}, \quad M^{\varepsilon}(R) \equiv \max _{B(R)} v^{\varepsilon}, \quad \underset{B(R)}{\operatorname{osc}} v^{\varepsilon} \equiv M^{\varepsilon}(R)-m^{\varepsilon}(R) .
$$

LEMMA 2.2. There exist a constant $C_{1}(\eta)=C_{1}(n, \theta, \Theta, M, C(\eta))$ and, for each $0<\alpha<1, a$ conitant $\gamma=\gamma(n, \theta, \Theta, \alpha)>0$ such that

(a) if $\Gamma \subset B(R)$ is closed and

$$
\text { meas } \Gamma \geqslant \alpha \text { meas } B(R),
$$

then

$$
C_{1}(\eta) R^{2}+m^{\varepsilon}\left(\frac{R}{4}\right) \geqslant \gamma \min _{\Gamma} v^{\varepsilon}+(1-\gamma) m^{\varepsilon}(R) .
$$

(b) In particular,

$$
\operatorname{meas}\left\{x \in B(R) \mid v^{\varepsilon} \geqslant \lambda m^{\varepsilon}(R)+(1-\lambda) M^{\varepsilon}(R)\right\} \geqslant \alpha \text { meas } B(R)
$$

implies

$$
C_{1}(\eta) R^{2}+m^{\varepsilon}\left(\frac{R}{4}\right) \geqslant \gamma(1-\lambda) \underset{B(R)}{\operatorname{osc}} v^{\varepsilon}+m^{\varepsilon}(R)
$$

for all $0<\lambda<1$. The various constants here do not depend on $\varepsilon, \xi$ or $R$, and only $C_{1}$ depends on $\eta$.

This lemma follows from Lemma 2.1 and [11]: see [4, §3] for details.

3. The main estimate. We are now ready to prove

Proposition 3.1. Let $u^{\varepsilon}$ be a smooth solution of $(2.2)_{\varepsilon}$, and assume that the estimate (2.3) holds. Then there exist a constant $\beta>0$ and, for each $\Omega^{\prime} \subset \subset \Omega$, a constant $C\left(\Omega^{\prime}\right)$ such that

$$
\left[D^{2} u^{\varepsilon}\right]_{C^{\beta}\left(\bar{\Omega}^{\prime}\right)} \leqslant C\left(\Omega^{\prime}\right) .
$$

The constant $\beta$ depends on $n, \theta, \Theta$, the coefficients of the operators $L^{k}$, and the $f^{k}$; $C\left(\Omega^{\prime}\right)$ depends only on these quantities and $\operatorname{dist}\left(\Omega^{\prime}, \partial \Omega\right)$ : neither depends on $\varepsilon$.

The proof will follow several preparatory lemmas:

LEMMA 3.2. There exist an integer $N \geqslant n$, unit vectors $\xi_{i}(i=1, \ldots, N)$ and constants $0<\theta^{\prime} \leqslant \Theta^{\prime}<\infty$ such that

$$
\beta_{\varepsilon}\left(L^{1} u^{\varepsilon}-f^{1}, \ldots, L^{m} u^{\varepsilon}-f^{m}\right) \equiv G^{\varepsilon}\left(u_{\xi_{1} \xi_{1}}^{\varepsilon}, \ldots, u_{\xi_{N} \xi_{N}}^{\varepsilon}, x\right)=0
$$

where $G^{\varepsilon}: \mathbf{R}^{n} \times \Omega \rightarrow \mathbf{R}$ is a smooth function, with

$$
\theta^{\prime} \leqslant G_{k}^{\varepsilon}(\cdot) \leqslant \Theta^{\prime} \quad(k=1,2, \ldots, N) .
$$

This lemma follows from considerations in $[4, \S 5]$. In particular we demonstrated there (following Kurtz [12]) that each operator $L^{k}$ can be rewritten in the form

$$
L^{k} u^{\varepsilon} \equiv \sum_{i=1}^{N} a_{i}^{k}(x) u_{\xi_{i} \xi_{i}}^{\varepsilon}+\sum_{i=1}^{n} b_{i}^{k}(x) u_{x_{i}}^{\varepsilon}(x)+c^{k}(x) u^{\varepsilon}(x)
$$


where the $\left\{\xi_{i}\right\}$ are some collection (independent of $k$ ) of unit vectors in $\mathbf{R}^{n}$, and the $a_{i}^{k}(x)$ are smooth functions,

$$
\theta^{\prime} \leqslant a_{i}^{k}(x) \leqslant \Theta^{\prime} \quad(i=1, \ldots, N ; k=1, \ldots, m ; x \in \Omega) .
$$

Now insert the representation (3.3) into the $\beta_{\varepsilon}$, recall (2.1)(ii) to check (3.2), and note that the lower order terms in (3.3) are Lipschitz according to (2.3).

Let us also note that we may arrange things so that the collection of unit vectors $\left\{\xi_{i}\right\}$ includes

$$
f_{i j}^{ \pm}=\frac{e_{i} \pm e_{j}}{\sqrt{2}} \quad(1 \leqslant i, j \leqslant n) .
$$

To see this fix $\delta>0$ so small that

$$
\tilde{L}^{k} u \equiv L^{k} u-\delta \sum_{i, j=1}^{n}\left(u_{f_{i j}^{+}, f_{i j}^{+}}+u_{f_{i j}^{-}, f_{i j}^{-}}\right)
$$

is uniformly elliptic and then apply the representation (3.3) to $\tilde{L}^{k}$ instead of $L^{k}$.

In light of all this there is a constant $C_{2}=C_{2}(n, m, N, M)$ such that if $B\left(R_{0}\right) \subset \Omega$ for some $R_{0}>0$, then

$$
\underset{B(R)}{\operatorname{Osc}} \sum_{i, j=1}^{n}\left(u_{x_{i} x_{j}}^{\varepsilon}-n^{2} M \delta_{i j}\right)^{2} \leqslant C_{2} \max _{1 \leqslant i \leqslant N} \underset{B(R)}{\operatorname{osc}} u_{\xi_{i} \xi_{i}}^{\varepsilon}
$$

for each $0<R<R_{0}$.

Then (3.5) implies

$$
\max _{1 \leqslant i \leqslant N} \underset{B(R)}{\operatorname{osc}} u_{\xi_{i} \xi_{i}}^{\varepsilon} \leqslant C_{3} \max _{1 \leqslant i \leqslant N} \underset{B(R)}{\operatorname{osc}} v_{i}^{\varepsilon}
$$

for some $C_{3}=C_{3}\left(C_{2}, n, m, N\right)$, provided $\eta>0$ is small enough; here

$$
v_{i}^{\varepsilon} \equiv u_{\xi_{i} \xi_{i}}^{\varepsilon}-\eta \sum_{i, j=i}^{n}\left(u_{x_{i} x_{j}}^{\varepsilon}-n^{2} M \delta_{i j}\right)^{2} .
$$

Our principal estimate is this (cf. [4, Lemma 4.3]):

Lemma 3.3. Assume that the ball $B\left(R_{0}\right) \subset \Omega$ for some $R_{0}>0$. If $\eta>0$ is small enough, there exist constants $\delta_{1}<1$ and $C_{4}$ such that if $0<R<R_{0}$, then

$$
\underset{B(R / 4)}{\operatorname{Osc}} v_{i}^{\varepsilon} \leqslant \delta_{1} \underset{B(R)}{\operatorname{OSc}} v_{i}^{\varepsilon}+C_{4} R
$$

for some index $i \in\{1, \ldots, N\}$. These constants do not depend on $R$ or $\varepsilon$.

Proof. As before we suppress the superscript " $\varepsilon$ " for notational simplicity;

$$
m_{i}(R) \equiv \min _{B(R)} v_{i}, \quad M_{i}(R) \equiv \max _{B(R)} v_{i} .
$$

There are two possibilities to consider:

Case I. Suppose

$$
\operatorname{meas}\left\{x \in B(R) \mid v_{i} \geqslant \lambda m_{i}(R)+(1-\lambda) M_{i}(R)\right\} \geqslant \operatorname{meas} B(R) / 2 N
$$


for some index $i \in\{1, \ldots, N\}$, say $i=1$, where $0<\lambda<1$ is a constant to be selected later. Then applying Lemma 2.2(b) to $v_{1}$ we find

$$
C_{1}(\eta) R^{2}+m_{1}\left(\frac{R}{4}\right) \geqslant \gamma(1-\lambda) \underset{B(R)}{\operatorname{osc}} v_{1}+m_{1}(R)
$$

for some fixed $\gamma>0$. Thus

$$
\begin{aligned}
\underset{B(R / 4)}{\operatorname{osc} v_{1}} & =M_{1}\left(\frac{R}{4}\right)-m_{1}\left(\frac{R}{4}\right) \leqslant M_{1}(R)-m_{1}\left(\frac{R}{4}\right) \\
& \leqslant(1-\gamma(1-\lambda)) \underset{B(R)}{\operatorname{osc}} v_{1}+C_{1}(\eta) R^{2} .
\end{aligned}
$$

This proves (3.8) with $\delta_{1}=(1-\gamma(1-\lambda))<1$.

Case II. Assume now that (3.9) fails for each $i=1,2, \ldots, N$. Then

$$
\text { meas } \Gamma_{1} \geqslant \frac{1}{2} \text { meas } B(R) \text {, }
$$

where

$$
\Gamma_{1} \equiv\left\{x \in B(R) \mid v_{i}<\lambda m_{i}(R)+(1-\lambda) M_{i}(R), i=1, \ldots, N\right\} .
$$

Let us suppose with no loss of generality

$$
\underset{B(R / 4)}{\operatorname{Osc}} v_{N}=\max _{1 \leqslant i \leqslant N} \underset{B(R / 4)}{\operatorname{osc}} v_{i} .
$$

Now (3.1) and (3.2) together imply that we can solve for $u_{\xi_{N} \xi_{N}}$ in terms of the other derivatives and $x$ :

$$
u_{\xi_{N} \xi_{N}}=g\left(u_{\xi_{1} \xi_{1}}, \ldots, u_{\xi_{N-1} \xi_{n-1}}, x\right)
$$

where $g: \mathbf{R}^{N-1} \times \Omega \rightarrow \mathbf{R}$ is smooth,

$$
-\frac{\Theta^{\prime}}{\theta^{\prime}} \leqslant g_{k} \equiv-\frac{G_{k}}{G_{n}} \leqslant-\frac{\theta^{\prime}}{\Theta^{\prime}} \quad(k=1,2, \ldots, N-1) .
$$

The function $g$ is thus strictly decreasing in its first $N-1$ arguments; and so for $x \in \Gamma_{1}$ we may estimate

$$
\begin{aligned}
u_{\xi_{N} \xi_{N}}(x) & =g\left(u_{\xi_{1} \xi_{1}}(x), \ldots, u_{\xi_{N-1} \xi_{N-1}}(x), x\right) \\
& =g\left(v_{1}(x)+\eta w(x), \ldots, v_{N-1}(x)+\eta w(x), x\right),
\end{aligned}
$$

where

$$
w \equiv \sum_{i, j=1}^{n}\left(u_{x_{i} x_{j}}-n^{2} M \delta_{i j}\right)^{2}
$$

Hence, since $x \in \Gamma_{1}$,

$$
\begin{aligned}
u_{\xi_{N} \xi_{N}}(x) & \geqslant g\left(\ldots, \lambda m_{i}(R)+(1-\lambda) M_{i}(R)+\eta w(x), \ldots, x\right) \\
& \geqslant g\left(\ldots, \lambda \min _{B(R)} u_{\xi_{i} \xi_{i}}+(1-\lambda) \max _{B(R)} u_{\xi_{i} \xi_{i}}, \ldots, x\right)-C \eta \underset{B(R)}{\operatorname{osc}} w,
\end{aligned}
$$


because

$\left|\lambda m_{i}(R)+(1-\lambda) M_{i}(R)+\eta w(x)-\left(\lambda \min _{B(R)} u_{\xi_{i} \xi_{i}}+(1-\lambda) \max _{B(R)} u_{\xi_{i} \xi_{i}}\right)\right| \leqslant \eta \underset{B(R)}{\operatorname{osc} w .}$

Therefore

$$
\begin{aligned}
u_{\xi_{N} \xi_{N}}(x) & \geqslant g\left(\ldots, \min _{B(R)} u_{\xi_{i} \xi_{i}}, \ldots, x\right)-C(\eta+(1-\lambda)) \max _{1 \leqslant i \leqslant N} \underset{B(R)}{\operatorname{osc}} u_{\xi_{i} \xi_{i}} \quad(\operatorname{see}(3.5)) \\
& \geqslant \max _{B(R)} u_{\xi_{N} \xi_{N}}-C R-C(\eta+(1-\lambda)) \max _{1 \leqslant i \leqslant N}^{\operatorname{osc} v_{B(R)}}
\end{aligned}
$$

by (3.6).

Hence for $x \in \Gamma_{1}$,

$$
\begin{aligned}
v_{N}(x) & =u_{\xi_{N} \xi_{N}}(x)-\eta w(x) \\
& \geqslant M_{N}(R)-C R-C(\eta+(1-\lambda)) \max _{1 \leqslant i \leqslant N} \underset{B(R)}{\operatorname{osc}} v_{i} .
\end{aligned}
$$

Next apply Lemma 2.2(a) to $v_{N}$ :

$$
\begin{aligned}
C(\eta) R^{2}+m_{N}\left(\frac{R}{4}\right) \geqslant & \gamma\left(M_{N}(R)-C R-C(\eta+(1-\lambda)) \max _{1 \leqslant i \leqslant N} \underset{B(R)}{\operatorname{osc}} v_{i}\right) \\
& +(1-\gamma) m_{N}(R) .
\end{aligned}
$$

From this we calculate

$$
\begin{aligned}
\underset{B(R / 4)}{\operatorname{osc}} v_{N} & =M_{N}\left(\frac{R}{4}\right)-m_{N}\left(\frac{R}{4}\right) \\
& \leqslant(1-\gamma+C(\eta+(1-\lambda))) \underset{1 \leqslant i \leqslant N}{\max } \underset{B(R)}{\operatorname{osc}} v_{i}+C R+C(\eta) R^{2} \\
& \leqslant \delta_{1} \max _{1 \leqslant i \leqslant N} \underset{B(R)}{\operatorname{osc}} v_{i}+C(\eta) R,
\end{aligned}
$$

with $\delta_{1}<1$, provided $\lambda<1$ is now fixed close enough to 1 and $\eta$ is small enough.

In view of (3.11), (3.8) is now proved for Case II.

The next lemma extends Lemma 3.3 by showing that if we pass from $B(R)$ to a (much) smaller ball, the sum of the oscillations of all the $v^{\varepsilon}$ diminishes by a fraction less than one, except for a correction term of order $R$.

LEMMA 3.4. There exist an integer $k_{N}$ and constants $\delta_{N}<1, C_{5}$ such that

$$
\sum_{i=1}^{N} \underset{B\left(R / 4^{k_{N}}\right)}{\operatorname{osc}} v_{i}^{\varepsilon} \leqslant \delta_{N} \sum_{i=1}^{N} \underset{B(R)}{\operatorname{osc}} v_{i}^{\varepsilon}+C_{5} R .
$$

These constants do not depend on $R$ or $\varepsilon$.

Proof. The argument is very similar to the proofs of $[4$, Lemmas $4.4,4.5]$ and so we only outline here the principal ideas. Once more the superscripts " $\varepsilon$ " are omitted.

First, owing to Lemma 3.3, the oscillation of at least one of the $v_{i}$, say $v_{1}$, diminishes by a fraction $\delta_{1}<1$ (and there is a correction term $C R$ ) if we pass from $B(R)$ to $B(R / 4)$ :

$$
\underset{B(R / 4)}{\operatorname{Osc}} v_{1} \leqslant \delta_{1} \underset{B(R)}{\operatorname{Osc}} v_{1}+C R
$$


Now consider $B(R / 4)$ and $B\left(R / 4^{2}\right)$. Again the oscillation of at least one of the $v_{j}$ decreases by the fraction $\delta_{1}$, and so there are two possibilities:

(a) If we can take $j \in\{2, \ldots, N\}$, say $j=2$, then a statement analogous to (3.14) obtains for $v_{2}$ and we may add this inequality to (3.14) to obtain an inequality of the type (3.13), but with the summation only from 1 to 2 .

(b) If on the other hand the oscillation of only $v_{1}$ diminishes in moving from $B(R / 4)$ to $B\left(R / 4^{2}\right)$, we may combine (3.14) and the same statement for the smaller ball to find

$$
\underset{B\left(R / 4^{2}\right)}{\operatorname{osc}} v_{1} \leqslant\left(\delta_{1}\right)^{2} \underset{B(R)}{\operatorname{osc}} v_{1}+C R .
$$

Now consider $B\left(R / 4^{2}\right)$ and $B\left(R / 4^{3}\right)$. If case (a) holds here we have (3.13) for $v_{1}$ and $v_{2}$; and case (b) on the other hand implies

$$
\underset{B\left(R / 4^{3}\right)}{\operatorname{osc}} v_{1} \leqslant\left(\delta_{1}\right)^{3} \underset{B(R)}{\operatorname{osc}} v_{1}+C R .
$$

Continuing this way, after finitely many steps we reach a ball $B\left(R / 4^{k_{2}-1}\right)$ by which either (3.13) obtains for $N=2$ or else inequalities like (3.15), (3.16) are valid, the latter possibility implying the oscillation of $v_{1}$ over $B\left(R / 4^{k_{2}-1}\right)$ is so negligible that an argument like that establishing Lemma 3.3 shows at the next step that the oscillation of some other $v_{i}(i \in\{2, \ldots, N\})$ must diminish. See [4, Lemma 4.4] for details.

Having so far proved (3.13) with 2 in place of $N$, we continue the process by induction eventually to arrive at (3.13) for $N$ itself: again we refer to [4, Lemma 4.5] for more explanation.

Proof of Proposition 3.1. A standard lemma in elliptic theory (cf. GilbargTrudinger [8, p. 191]) states that Lemma 3.4 implies

$$
\underset{B(R)}{\operatorname{Osc}} v_{\varepsilon}^{i} \leqslant C\left(\frac{R}{R_{0}}\right)^{\beta} \quad(i=1, \ldots, N)
$$

for certain numbers $C>0,0<\beta<1$; these constants depend only on known quantities. We apply this calculation to any ball $B\left(x_{0}, R_{0}\right), x_{0} \in \bar{\Omega}^{\prime} \subset \subset \Omega, R_{0}<$ $\operatorname{dist}\left(x_{0}, \partial \Omega\right)$ to complete the proof.

4. Proof of Theorem 1. It is now fairly easy to exploit the a priori estimates from $\S 3$ in proving an existence, regularity, and uniqueness theorem for the HamiltonJacobi-Bellman equation. First we solve (2.2) $)_{\varepsilon}$ and will then pass to limits as $\varepsilon \searrow 0$.

As previously noted we do not have a priori Hölder estimates for $D^{2} u$ near $\partial \Omega$, and so first we must modify the operators $L^{k}$ to be identical near the boundary.

Define for $\delta>0$

$$
\Omega_{\delta} \equiv\{x \in \Omega \mid \operatorname{dist}(x, \partial \Omega)>\delta\},
$$

and next choose a smooth cutoff function $\zeta=\zeta_{\delta}$ such that

$$
0 \leqslant \zeta \leqslant 1, \quad \zeta \equiv 1 \text { on } \Omega_{\delta}, \quad \zeta \equiv 0 \text { near } \partial \Omega \text {. }
$$

Now define

$$
L_{\delta}^{k} u \equiv(1-\zeta) \theta \Delta u+\zeta L^{k} u \quad(k=1,2, \ldots, m)
$$


these operators are uniformly elliptic, with smooth coefficients. We also set

$$
f_{\delta}^{k}=\zeta f^{k} \quad(k=1,2, \ldots, m) .
$$

In view of (4.1) and (2.1)(iv)

$$
\beta^{\varepsilon}\left(\ldots, L_{\delta}^{k} u-f_{\delta}^{k}, \ldots\right)=\theta \Delta u \text { for } x \text { near } \partial \Omega .
$$

Hence Proposition 3.1 and the standard boundary Schauder estimates provide the $a$ priori estimate

$$
\left[D^{2} u_{\delta}^{\varepsilon}\right]_{C^{\beta}(\bar{\Omega})} \leqslant \text { Const. }
$$

(for some $\beta>0$ ), where $u_{\delta}^{\varepsilon}$ is a smooth solution of

$$
\begin{cases}\beta^{\varepsilon}\left(\ldots, L^{k} u_{\delta}^{\varepsilon}-f_{\delta}^{k}, \ldots\right)=0 & \text { in } \Omega, \\ u_{\delta}^{\varepsilon}=0 & \text { on } \partial \Omega .\end{cases}
$$

The bound in (4.3) requires a previous estimate (cf. (2.3))

$$
\max _{x \in \Omega}\left|u_{\delta}^{\varepsilon}\right|,\left|\dot{D} u_{\delta}^{\varepsilon}\right|,\left|D^{2} u_{\delta}^{\varepsilon}\right| \leqslant M_{\delta},
$$

but this follows from fairly straightforward modifications of the techniques in Evans-Lions [6].

It is now routine to prove the existence of a unique, smooth solution of $(4.4)_{\varepsilon, \delta}$ using a standard continuation argument. The solution belongs to $C^{2, \beta}(\bar{\Omega})$ and so customary bootstrap-type arguments demonstrate that $u_{\varepsilon}^{\delta}$ is in fact smooth.

Next we send $\delta \searrow 0$ to solve (2.2) . For this it suffices to note that

$$
\beta^{\varepsilon}\left(\ldots, L^{k} u_{\varepsilon}^{\delta}-f^{k}, \ldots\right)=0 \text { in } \Omega_{\delta} .
$$

Now an estimate like that used for [4, Lemma 2.2] proves

$$
\max _{\Omega}\left|u_{\varepsilon}^{\delta}\right| \leqslant C_{6},
$$

the constant independent of $\varepsilon$ and $\delta$. From this bound and interior estimates (cf. Lenhart [15]) of the form

$$
\max _{\Omega^{\prime}}\left|D u_{\varepsilon}^{\delta}\right|,\left|D^{2} u_{\varepsilon}^{\delta}\right| \leqslant C_{7}\left(\Omega^{\prime}\right)
$$

(for $\Omega^{\prime} \subset \subset \Omega_{\delta} \subset \subset \Omega ; C_{7}\left(\Omega^{\prime}\right)$ independent of $\varepsilon, \delta$ ), we may recall Proposition 3.1 and extract a subsequence of the $u_{\varepsilon}^{\delta}$ converging in $C^{2, \alpha}(\Omega)(0<\alpha<\beta)$ as $\delta \searrow 0$ to a function $u^{\varepsilon}$ solving

$$
\beta_{\varepsilon}\left(\ldots, L^{k} u^{\varepsilon}-f^{k}, \ldots\right)=0 \text { in } \Omega
$$

Furthermore

$$
u^{\varepsilon} \in C(\bar{\Omega}), \quad u^{\varepsilon}=0 \quad \text { on } \partial \Omega
$$

this follows since we can find (as in [4, Lemma 2.2]) a smooth barrier function $\Phi$ such that

$$
\begin{cases}-\Phi \leqslant u_{\varepsilon}^{\delta} \leqslant \Phi & \text { in } \Omega \\ \Phi=0 & \text { on } \partial \Omega\end{cases}
$$


for all $\varepsilon, \delta$. Hence $u^{\varepsilon}$ solves (2.2) $)_{\varepsilon}$ and-from (4.7)-(4.9)-we have

$$
\left\{\begin{array}{l}
\max _{\Omega}\left|u^{\varepsilon}\right| \leqslant C_{6}, \\
\max _{\Omega^{\prime}}\left|D u^{\varepsilon}\right|,\left|D^{2} u^{\varepsilon}\right| \leqslant C_{7}\left(\Omega^{\prime}\right), \\
-\Phi \leqslant u^{\varepsilon} \leqslant \Phi \quad \text { in } \Omega .
\end{array}\right.
$$

Recalling Proposition 3.1 once more we pass to limits as $\varepsilon \searrow 0$ to construct a solution $u \in C(\bar{\Omega}) \cap C^{2, \beta}(\Omega)$ of the Hamilton-Jacobi-Bellman equation. Uniqueness is a consequence of the maximum principle.

NoTE ADDED IN PROOF. N. S. Trudinger has recently greatly simplified the proofs in this paper and in [4]: see the forthcoming second edition of [8] for this.

\section{REFERENCES}

1. S. Belbas, Weak solutions of the Hamilton-Jacobi-Bellman equation, preprint.

2. A. Bensoussan and J. L. Lions, Applications des inéquations variationelles en contrôle stochastique, Dunod, Paris, 1979.

3. H. Brezis and L. C. Evans, A variational inequality approach to the Bellman-Dirichlet problem for two elliptic operators, Arch. Rational Mech. Anal. 71 (1979), 1-14.

4. L. C. Evans, Classical solutions of fully nonlinear, convex, second order elliptic equations, Comm. Pure Appl. Math. 35 (1982), 333-363.

5. L. C. Evans and A. Friedman, Optimal stochastic switching and the Dirichlet problem for the Bellman equation, Trans. Amer. Math. Soc. 253 (1979), 365-389.

6. L. C. Evans and P. L. Lions, Résolution des équations de Hamilton-Jacobi-Bellman, C. R. Acad. Sci. Paris 290 (1980), 1049-1052.

7. W. Fleming and R. Rishel, Deterministic and stochastic optimal control, Springer, New York, 1975.

8. D. Gilbarg and N. S. Trudinger, Elliptic partial differential equations of second order, Springer, New York, 1977.

9. N. V. Krylov, Controlled diffusion processes, Springer, New York, 1981.

10. Control of a solution of a stochastic integral equation, Theor. Probab. Appl. 17 (1972), 114-130.

11. N. V. Krylov and M. V. Safonov, An estimate of the probability that a diffusion hits a set of positive measure, Soviet Math. 20 (1979), 253-256.

12. T. Kurtz, Representations of Markov processes as multiparameter time changes, preprint.

13. H. Kushner, Stochastic stability and control, Academic Press, New York, 1967.

14. O. A. Ladyženskaja and N. N. Ural'ceva, Linear and quasilinear elliptic equations, Academic Press, New York, 1968.

15. S. Lenhart, Ph.D. Thesis, University of Kentucky, 1981; Indiana Univ. Math. J. (to appear).

16. P. L. Lions, Résolution analytique des problèmes de Bellman-Dirichlet, Acta. Math. 146 (1981), 151-166.

17. __ Thèse d'État, Paris VI, 1979.

18. P. L. Lions and J. L. Menaldi, Optimal control of stochastic integrals and Hamilton-Jaocbi-Bellman equations I, II, SIAM J. Control and Optim. 20 (1982), 58-95.

19. M. Nisio, Remarks on stochastic optimal controls, Japan J. Math. 1 (1975), 159-183.

Department of Mathematics, University of Maryland, College Park, Maryland 20742 